\title{
ANTROPOLOGÍA
}

\section{COMPETENCIA CULTURAL EN ENFERMERÍA: POBLACIÓN SUBSAHARIANA}

\author{
Isabel Gentil García
}

Doctora. Profesora E. U. Enfermería, Fisioterapia y Podología. Universidad Complutense de Madrid

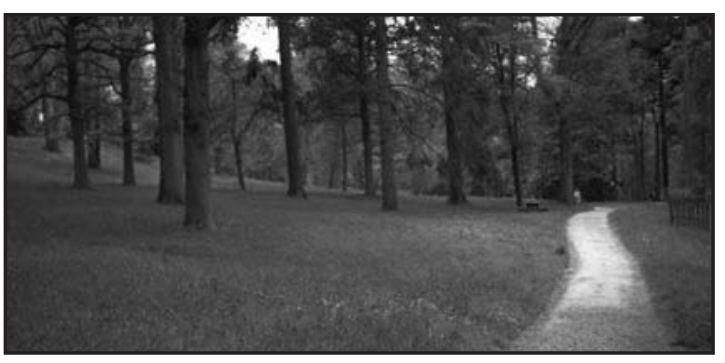

THE CULTURAL COMPETENCE IN THE NURSING PROFESSIONAL: SUB-SAHARAN POPULATION

\section{SUMMARY}

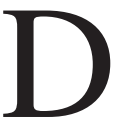

eparting from the widespread idea of the need of intercultural mediators in the sanitary world as the solution to the derivative difficulties of working with persons of other cultures, this work has as aim demonstrate that the necessary thing is that the professionals of nursing we form ourselves to realize taken care culturally competent. There are analyzed certain premises without which they cannot be realized taken care competent. The fundamental thing is to have a constructive attitude towards the cultural differences and sensibility towards the persons of other cultures. Also it is necessary increase the knowledge on other cultures. By it values and beliefs are described in relation to health and disease in subSaharan population. Coming to the conclusion that the human beings though diverse culturally we share many common features in relation to the disease.

Key words: Cultural competence. Nursing. Health. Immigration. Population Subsahariana.
COMPETÊNCIA CULTURAL NA ENFERMAGEM: POPULAÇÃO SUBSAHARIANA

\section{RESUMO}

1 artindo da ampla ideia da necessidade de mediadores interculturais no mundo da saúde como a solução para as dificuldades derivadas do trabalho com pessoas de outras culturas, este estudo teve como objetivo demonstrar que é necessário que os profissionais de enfermagem sejam formados para realizar cuidados culturalmente competentes. O fundamental é ter uma atitude construtiva em direção às diferenças culturais e a sensibilidade em relação às pessoas de outras culturais. Também se faz necessário aumentar os conhecimentos sobre outras culturas. Por essa razão se descrevem valores e crenças em relação à saúde e à doença na população subsahariana. Chegamos à conclusão de que os seres humanos, ainda que diversos culturalmente, compartilhamos muitos aspectos em relação ao adoecimento.

Palavras chave: Competência cultural. Enfermagem. Saúde. Imigração. População Subsahariana.

\section{RESUMEN}

0 artiendo de la extendida idea de la necesidad de mediadores interculturales en el mundo sanitario como la solución a las dificultades derivadas de trabajar con personas de otras culturas, este trabajo tiene como objetivo demostrar que lo necesario es que los profesionales de enfermería nos formemos para realizar cuidados culturalmente competentes. Se analizan ciertas premisas sin las 
cuales no se pueden realizar cuidados competentes. Lo fundamental es tener una actitud constructiva hacia las diferencias culturales y sensibilidad hacia las personas de otras culturas. También se necesita aumentar los conocimientos sobre otras culturas. Por ello se describen valores y creencias en relación a salud y enfermedad en población subsahariana. Llegando a la conclusión que los humanos aunque diversos culturalmente compartimos muchos rasgos comunes en relación a la enfermedad.

Palabras clave: Competencia cultural. Enfermería. Salud. Inmigración. Población Subsahariana.

\section{INTRODUCCIÓN}

Los dinámicos profesionales de Enfermería conscientes de que el porcentaje de población extranjera en nuestra sociedad es de $12 \%$ (MTI, 2009) y que sus lugares de procedencia son los cinco continentes han realizado y publicado numerosos estudios sobre cómo mejorar los cuidados de acuerdo a esta nueva situación social multicultural. La mayoría de los estudios llegan a la conclusión de la necesidad de mediadores interculturales en el mundo sanitario. Creo que es un error. Se hace referencia a ellos como la panacea universal a las dificultades derivadas de trabajar con personas de otras culturas.

Este artículo tiene como objetivo demostrar que lo realmente necesario es que los profesionales de enfermería nos formemos para realizar cuidados culturalmente competentes. Está estructurado en tres partes: Definición de conceptos y reflexión sobre competencia cultural, trabajo de campo con población subsahariana y conclusiones.

\section{¿ES NECESARIO EL MEDIADOR INTER- CULTURAL EN ENFERMERÍA?}

Solicitar mediadores interculturales implica un delegar, una pasividad, un desprenderse de responsabilidades profesionales. Distinto es el problema de la barrera lingüística, aquí sí puede ser necesaria la presencia de un traductor.

La solución ante nuevas situaciones y nuevos retos en el ámbito profesional es formarse. En estos momentos hemos incorporado la informática como una herramienta más de nuestro trabajo. $\mathrm{Si}$ unos años atrás cuando ello se inició hubiéramos optado por proclamar la necesidad de "mediadores informáticos", hoy seríamos incompetentes para nuestro trabajo. Lo que hay que reclamar es formación para ser "competentes culturalmente", como un elemento más de nuestra competencia profesional.

Todavía más importante: mientras reclamemos la presencia de mediadores interculturales mantendremos, sin pretenderlo, en situación de exclusión al colectivo cultural diferente.

Conocer otros conceptos de salud y enfermedad, hábitos dietéticos, creencias religiosas, mitos, costumbres, conductas... es adaptarnos a nuevas necesidades, centrándonos en la persona enferma más que en la enfermedad. Somos los profesionales de enfermería los que más entendemos que no hay cuerpo-máquina enfermo, sino personas con enfermedad, por esto debemos orientarnos hacia el cuidado cultural competente.

\section{CUIDADO CULTURAL COMPETENTE}

Numerosos profesionales de Enfermería que nos han precedido en tiempos históricos lo han practicado, aunque no le dieran este nombre.

En los años 50 del siglo pasado, Madeleine Leininger, enfermera y antropóloga, se anticipó a Organismos Internacionales para elaborar el concepto (Siles, 2001). Leininger considera que la competencia cultural es un imperativo para las enfermeras si queremos realizar nuestra función con efectividad. Entendió que sumar cuidados y sociedad multicultural daba como resultado Enfermería Transcultural (ETC), definiéndola como el área formal de estudio y trabajo centrado en el cuidado basado en la cultura, creencias de salud o enfermedad, valores y prácticas de las personas, para ayudarlas a mantener o recuperar su salud, hacer frente a sus discapacidades o a su muerte. El objetivo de ETC es proporcionar cuidados culturalmente competentes, (Leininger, 1999)

En los años 80, la OMS reconoce la importancia de la cultura y de la diversidad cultural en el proceso salud-enfermedad y se plantea cómo mejor atender sanitariamente a las minorías étnicas con diferentes culturas y lenguas, (Herman, 2007) 
En 2007, el Panel de Expertos en Competencia Cultural de la Academia Americana de Enfermería define Competencia Cultural como: "Tener conocimiento, actitudes y habilidades sobre grupos culturales diversos que permite al profesional de la salud proporcionar cuidado cultural de acuerdo a las necesidades manifestadas por el paciente. Es un proceso continuo que involucra la aceptación y el respeto de las diferencias y no permite que las creencias personales propias tengan una excesiva influencia en aquellos que tienen una visión del mundo diferente de la propia”, (Giger, 2007).

\section{¿QUÉ NECESITO SABER PARA SER CUL- TURALMENTE COMPETENTE?}

Es necesario tener conocimientos sobre otras culturas, pero no es necesario conocer todas las tradiciones culturales de todas las personas sanas y enfermas con que interactúo. También en nuestra sociedad de procedencia existe la diversidad.

Debo saber que la percepción de enfermedad, las causas de la misma, las actitudes hacia ella, los métodos para tratarla y los cuidados están influidos culturalmente, pero siempre están íntimamente integrados en un marco mayor: el contexto social y cultural que determinada una cosmovisión, una forma de percibir el mundo.

Debo saber que las formas de entender los roles familiares y de parentesco en relación a la persona enferma están influenciadas culturalmente.

Debo saber que para dar cuidados culturalmente competentes es más importante tener una actitud constructiva hacia las diferencias culturales y sensibilidad hacia las personas de otras culturas.

Debo saber que sin estas premisas no conseguiré realizar cuidados competentes:

- Respeto. Las personas esperan de los profesionales de enfermería que entiendan y respeten las creencias y valores culturales que son fundamentales en su construcción como persona. No caer en el desprecio. Percibir el respeto es sentirse valorado. Sentirse valorado y respetado es la llave que abre la puerta a la confianza.

- Confianza. Generar confianza para facilitar la colaboración. La confianza no es lo mismo que una excesiva familiaridad. La confianza se genera mostrando interés por su problema de salud pero desde el rol profesional. Además escuchando, para comprender, no para juzgar.

Alcanzada la confianza no será raro que nos confíen los remedios tradicionales que están utilizando, generalmente infusiones u otros. Si es posible mantenerlos. Si no es posible y para que sigan los consejos de cuidados que les transmitamos, el mensaje pedagógico será, con argumentos razonados, enfatizando la mejora de su salud, no transmitirlo como prohibiciones y negaciones de su capital cultural. Se rechazará.

- Saber dialogar. El diálogo es la herramienta para alcanzar acuerdos. Dialogar es escuchar los argumentos del otro para tratar de comprender su perspectiva, contestar con la explicación desde nuestra perspectiva, reconociendo las similitudes y las diferencias y buscar-negociar la mejor solución para la persona enferma. Asumiendo que en ambos lados existen puntos e intereses que son comunes. Dialogando desde la igualdad de posiciones, no desde la superioridad.

- Valorar la diversidad cultural como un bien. La diversidad siempre es fructífera, productiva, generativa. Aprender a apreciar los valores y significados que encierran otras creencias que nos resultan extrañas. Todo el saber tradicional de otras culturas que ha ayudado a sobrevivir a los miembros de otras sociedades tiene algo importante que comunicarnos. Ver esa diversidad como la ocasión para el diálogo. No ver las diferencias culturales como enfrentamientos, porque entonces, lógicamente, tiene que haber ganadores y perdedores. No pretendamos ganar.

- Desarrollar actitudes que nos permitan comprender otros modelos explicativos de la enfermedad. La enfermedad no es sólo los signos, además es un conjunto de síntomas subjetivos, vivencia individual de esa enfermedad, que se construye con las experiencias y narraciones colectivas de una sociedad determinada. Puede suceder que las sensaciones corporales se interpreten como síntomas si así se ha aprendido en el contexto sociocultural o al contrario, lo que reconocemos como síntomas se desprecien como sensaciones corporales normales. El "síntoma" no es neutro, es etiquetado como enfermedad según el saber médico-cultural de cada sociedad.

- Huir de los estereotipos. Podemos creernos que somos objetivos y que vemos la "realidad" tal 
y como es, pero puede suceder que sean nuestros estereotipos mentales los que dibujan una realidad que sólo existe en nuestra mente. Por eso es importante sinceridad para reconocerlos y que no tiñan la percepción. (Gentil, 2006)

- Contribuir a disminuir la angustia de la persona derivada de estar enferma. Para ello no mostrar prisa, aunque el tiempo en nuestro sistema de salud es limitado. Saber que el concepto tiempo en sociedades más tradicionales es muy diferente al de las sociedades industrializadas donde el reloj impone los ritmos de trabajo, de ocio etc. Para ellos el tiempo está para el servicio de las personas, no las personas al servicio del tiempo. Saber que no siempre contestarán rápidamente a nuestras preguntas. El interrogatorio clínico de pregunta-breve respuesta-breve, no se entiende muy bien fuera de nuestra cultura occidental.

- Desarrollar el aprecio por los demás, el afecto, la compasión, la empatía. Empatía como la entiende Edith Stein "aprensión de las vivencias ajenas, apercibimiento del vivenciar de otro" (Nogales, 2008)

\section{DESCENDER DE LA TEORÍA A LOS CASOS: EVIDENCIAS EN POBLACIÓN SUBSAHARIANA}

(Es parte de un trabajo de investigación que está en proceso titulado: "Estudio en población inmigrante africana atendida en el sistema sanitario español. Necesidades de salud autopercibidas" que tiene como objetivo: Identificar valores, creencias, dificultades, necesidades, percepción sobre salud y enfermedad, en población inmigrante, a través de los relatos de su experiencia vivida al ser atendidos de alguna enfermedad para diseñar y practicar cuidados culturalmente adaptados. Metodología cualitativa con entrevistas en profundidad. Aprobado por el Departamento de Enfermería de la UCM. Investigadora principal, Isabel Gentil)

La población subsahariana no es la población inmigrante más numerosa (MTI, 4’12\%) pero quizá con la que sentimos mayor distancia cultural. Cuando nos referimos a población subsahariana incluimos población de varios países y con numerosas etnias en cada país, aun así, hay rasgos culturales que son compartidos.

La cosmovisión en la que se inscriben creencias y valores en África es sobre todo la tradición animista. Esta cosmovisión se transmite de forma oral, no escrita, a través de la repetición de los rela- tos de los más ancianos. Se interioriza en el proceso de enculturación y se refuerza durante toda la vida, pues en la escucha de los relatos tradicionales participan todos como oyentes. Es un conocimiento ancestral y compartido. "Desengáñate Isabel, en África hay musulmanes, pero son animistas, hay cristianos, pero son animistas, hay curas cristianos, pero son animistas, hay obispos cristianos, pero son animistas" (Informante Camerún)

En África las religiones de libro fueron introducidas e impuestas a la población siempre por los colonizadores, muchas veces por la fuerza, y fueron aceptadas en un sincretismo con el animismo. Aunque a algunos esta denominación les molesta. "No me gusta llamarla animismo, me gusta decir la religión tradicional africana" (Informante Benín). En estos momentos hay una reivindicación de las religiones y cultos tradicionales africanos. Más que reivindicación es un destapar lo que siempre permaneció presente y arraigado en sus corazones pero por la dominación colonial hubo que mantener oculto.

\section{Medicina tradicional africana}

Los saberes ancestrales sobre enfermedades y sus tratamientos forman un corpus de conocimientos muy valorado entre las personas de África, lo perciben como auténtica medicina. "Yo la llamo medicina tradicional africana. Utiliza mucho las plantas y la naturaleza, porque en la naturaleza está el remedio para todas las enfermedades." (Informante Benín). "El que conoce la naturaleza conoce sus misterios y los misterios de las enfermedades y el poder de la naturaleza para vencer las enfermedades. Aqui nadie tiene tiempo para escuchar a la naturaleza, ella habla y los sanadores africanos saben escucharla" (Informante Guinea Bissau)

Desde su cosmovisión hay una comunicación entre todo lo vivo (humanos, animales, plantas y naturaleza) porque todos comparten un mismo principio vital que lo integra todo. La salud es la relación armónica de todo ello. La enfermedad afecta no sólo a la persona que la padece sino a todo ese conjunto armónico de lo vivo, pero sobre todo a la comunidad de personas. De tal forma que la enfermedad en una persona es síntoma de desar- 


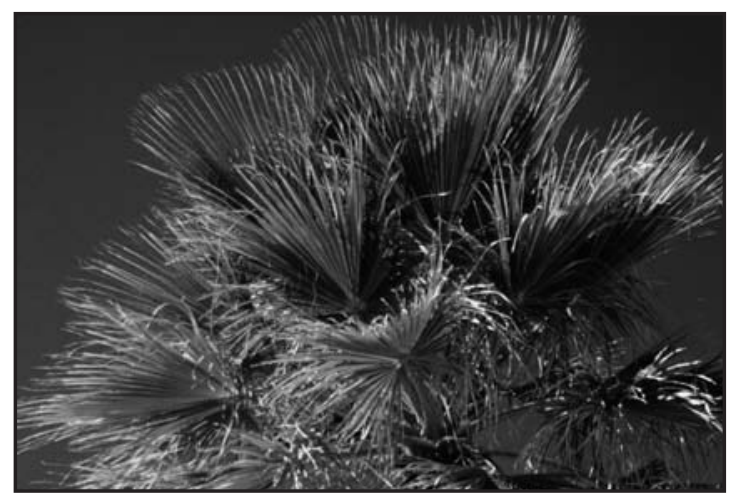

monía del grupo, Por ello el sanador pregunta al enfermo "¿piensa si hay algo o alguien detrás de tu enfermedad?"

\section{Conocer la "causa verdadera" de la enferme- dad}

Toda persona subsahariana enferma, independientemente de su etnia, necesita saber la "causa verdadera" de su enfermedad. El diagnóstico de la biomedicina occidental se respeta y se acepta, pero es totalmente insuficiente para su tranquilidad espiritual. Necesitan saber ¿quién o qué hizo que tenga esta enfermedad? "Sí, se tiene confianza en los profesionales sanitarios europeos, nos lo han trasmitido culturalmente: ellos te van a ayudar, pero... en cuanto puedes vas a recurrir a un sanador para que te diga la causa verdadera de la enfermedad. Nuestra mentalidad es que la enfermedad la provocan terceros. Siempre hay alguien o algo detrás: brujería, mal de ojo... Siempre, aunque sea una herida porque te has caído. Y necesitas saber la causa verdadera, es por tu tranquilidad". (Informante Guinea Bissau).

El concepto de salud es un concepto holístico y dan mucha importancia al aspecto espiritual. El bienestar psíquico es fundamental para sentirse con salud. "Es una necesidad básica consultar al sanador. Necesitas tranquilizarte. Necesitas seguridad. Siempre te pregunta ¿tienes problemas con alguien que te pudo hacer eso? Si dices que no luego se buscan otros caminos, pero sales sabiendo la causa verdadera de la enfermedad y eso te tranquiliza mucho" (Informante Ghana)

Esa búsqueda de la causa detrás de la causa no implica fatalismo e inacción, como pudiera deducirse de una interpretación apresurada, sino todo lo contrario implica capacidad de intervención para evitarlo y reflexión sobre la importancia de las buenas relaciones interpersonales en el grupo y sociedad.

\section{El curandero africano}

Los curanderos africanos son maestros en conocer el psiquismo de las personas. Han escuchado, conocido y meditado sobre tantos problemas y angustias de los humanos que se han convertido en auténticos sabios de la aflicción humana. Han sido testigos de tantas soluciones a problemas semejantes que pueden aconsejar con conocimiento. "El curandero africano te pregunta por tus problemas y quiere conocerlos y tu le cuentas todos tus malestares y qué te pasa y qué problemas tienes y qué no estás bien. El quiere saberlo todo de ti y luego no te da consejos directos: <haz esto>, sino que te habla con metáforas y tú mismo descubres qué tienes que hacer" (Informante Benín)

El curandero africano ha aprendido las enseñanzas de otro maestro. Se suele escoger para iniciar a un descendiente, observando primero el que parece tener más cualidades. Otras veces es alguien que se siente llamado por un antepasado para ser curandero. El sanador que hará de maestro le somete a unas pruebas antes de aceptarle. Siempre debe ser persona honesta. Poco a poco le irá trasmitiendo los secretos de la naturaleza. Esta enseñanza dura años. "En África hay muy buenos herboristas que preparan ungüentos, jarabes, infusiones con plantas y curan muchas enfermedades, el problema es que las plantas al traerlas aquí pierden su poder" (Informante Ghana).

\section{La comunicación}

La comunicación con el sanador africano es una conversación donde el enfermo no sólo informa escuetamente del síntoma sino que además manifiesta sus sentimientos de ansiedad, malestar, inquietud, desasosiegos etc. Habla de su padecimiento. Y refieren sus sensaciones emocionales entrelazadas con los síntomas somáticos porque no separan soma de psique. "Aquí tienes mucho estrés, en África no hay estrés. Si lo tienes vas a un curandero y él te cuenta mediante parábolas experiencias de vida (aunque sea analfabeto), te va relatando historias semejantes a la tuya y con ello 
te va trasmitiendo las posibles soluciones y sales tranquilo y contento y con solución a tu problema" (Informante Guinea Bissau)

La sabiduría ancestral africana está repleta de fábulas y alegorías que encierran enseñanzas prácticas, realzan valores de solidaridad y rechazan la maldad de comportamiento como el egoísmo. Son relatos orales que enseñan a vivir y a solucionar problemas.

Aquí, la demanda de comunicación con los profesionales de la salud es muy alta, la necesidad de comunicar queda frustrada en la medicina occidental. "Cuando voy a la consulta del médico tengo sed de diálogo y cuando salgo de la consulta aún tengo más sed" (Informante Benín)

\section{El tratamiento}

"Hay problemas que la medicina occidental no sabe curar" (Informante Ghana).

El tipo de problemas a que se refiere son trastornos psicosomáticos que se producen especialmente en personas que viven en situaciones de incertidumbre duraderas. $\mathrm{Y}$ que Atxtegui (Atxtegui, 2002) describe bien pero que muchas veces nuestra medicina de la "falta de tiempo" despacha con un antiansiolítico que pretende tratar el efecto obviando las causas.

Para la sanación del malestar deben enfrentarse dos polos contradictorios. En uno, la incertidumbre derivada de la enfermedad y el padecimiento. En el otro, la seguridad en la sabiduría del sanador. La plena confianza en la sabiduría del sanador y sus consejos es fundamental en el proceso de curación.

El tratamiento de la biomedicina occidental se sigue con confianza, se reconocen sus altos grados de conocimiento. "Por suerte gozo de buena salud y tengo médico de salud en España. La asistencia médica aquí no se puede comparar con mi país, es mejor incluso que en Estados Unidos. Aquí no tendría miedo de ingresar en un hospital, sí en mi país porque hay escasez de todo" (Informante Uganda).

Pero manifiestan que esta medicina occidental no cura el espíritu. Los sentimientos de inseguridad y desasosiego se suavizan con el sanador tradicional capaz de escuchar y orientar, con quien se puede hablar largamente de lo que inquieta. Aquí les falta ese tipo de sanador. "El problema es que aquí no han venido los curanderos más sabios, siguen viviendo en África. Aqui han venido los "buscavidas" les llamo yo. Han venido lo peor. Sólo lo hacen por dinero. Quieren aprovecharse, sin conocimiento. En África no pasa porque hay un colectivo de sabios que tienen un riguroso control y al farsante se le castiga fuerte." (Informante Guinea Bisau).

\section{El rol familiar con el enfermo}

La enfermedad es el elemento extraño que rompe la coherencia de un mundo armónico. La enfermedad no solo es ruptura de armonía con la naturaleza, también de la armonía en la comunidad de personas, pues las relaciones sociales son parte integrante del todo armónico, por ello en el tratamiento entra en juego las relaciones con el grupo, para reforzarlas. "Cuando estás enfermo el papel de la familia es fundamental. Hay una enorme cola de visitas. Aunque estés trabajando coges permiso $y$ además te lo dan. Y si algún familiar no va es motivo de distanciamiento”. (Informante Benín) "La cantidad de gente que viene a verte cuando estás enfermo es enorme, viene toda tu familia y tus amigos. Vienen para decirte que te aprecian y que debes curarte porque ellos también sufren con tu enfermedad". (Informante Uganda)

En estas sociedades cuando alguien está enfermo los miembros de esa sociedad se movilizan priorizando las necesidades de acompañamiento al enfermo sobre los intereses individuales. Algo así sucede con las personas españolas de etnia gitana. Cuando una persona ingresa en un hospital implica que la persona está en un momento especialmente lábil de su existencia, por ello todos los miembros del colectivo se movilizarán paralizando su actividad para acompañar al enfermo. La vida individual entra en pausa y el movimiento sólo está alrededor del enfermo y de la familia más próxima.

El concepto de familia africana no es el de familia nuclear, demasiado reducido para ellos. El concepto de familia es familia extensa con lazos muy fuertes con familiares que según nuestra nomenclatura de familia serían de segundo o tercer grado y que ellos utilizan para esos familiares el vocablo hermano y con el mismo significado de relación de afecto que le damos nosotros. Además en varios lugares existe el matrimonio poligínico por tradición. 
Cada sociedad ordena jerárquicamente sus valores. Hay sociedades que dan más importancia al individualismo (cultura occidental) y otras al colectivismo (culturas tradicionales). Las sociedades más tradicionales suelen ser más colectivistas, emocionalmente están más ligados al grupo y su comportamiento tiende a priorizar el interés por el grupo. La solidaridad es un valor importante en la sociedad africana. "Es cuando caes enfermo cuando sabes lo que te aprecian tus compañeros de trabajo”. (Informante Guinea Bissau).

\section{CONCLUSIÓN. LOS HUMANOS NO TAN DIFERENTES AUNQUE CON DIFERENTES CULTURAS}

La enfermedad es un universal en la humanidad. Todas las culturas buscan explicar el por qué de la enfermedad. La búsqueda de las causas naturales que originan las enfermedades es el motor de nuestro modelo científico. En culturas más tradicionales las personas buscan las causas en algo que le es cercano a su cosmovisión: fuerzas mágicas, brujería. Pero también en nuestra cultura hablamos de "mala suerte" (concepto mágico) de tal persona por haber cogido tal enfermedad.

Todas las sociedades tienen un lazo que liga la causa de la enfermedad con la sociedad y los comportamientos. Desde la biomedicina para las enfermedades cardiovasculares, el cáncer, la gripe y desde el animismo para "el mal de ojo", el "susto", la brujería. Es iluso pretender desligar la enfermedad del contexto social.

La enfermedad es uno de los problemas fundamentales para todas las sociedades, por ello todas las sociedades tienen una larga historia de búsqueda de remedios para tratar la enfermedad y el sufrimiento. Todos realizamos un sincretismo entre la biomedicina científica y las creencias populares tradiciones populares buscando paliar el sufrimiento y la curación (Pascual Martínez R., 2001). Y es que son en los momentos críticos, frágiles, vulnerables de nuestra vida, como pasar por una enfermedad, cuando más necesitamos aferrarnos a lo conocido, a aquello que más nos incrusta con aquellas creencias y valores más antiguos, aquellas que nos ayudaron en la infancia a constituirnos como personas.

Todas las sociedades tienen sanadores. En unas sociedades actúan desde el conocimiento científi- co, cuyos logros son indiscutibles, y en otras desde el conocimiento derivado de la observación y la experiencia, es decir desde el intelecto, no desde la irracionalidad.

Las personas subsaharianas perciben la medicina occidental con respeto y admiración para tratar las enfermedades pero la encuentran deficitaria en relación a la atención a la persona en su totalidad. No difiere mucho de la percepción de la población autóctona.

Aunque los médicos de nuestra biomedicina son respetados y admirados, son los profesionales de Enfermería a los que se les percibe como más humanos, más cercanos a sus necesidades. Los médicos aquí son muy profesionales, pero la gente más cercana, más humana con el enfermo es la enfermera, más que el médico. Lo contrario de lo que yo conozco de África" (Informante Ghana)

\section{BIBLIOGRAFÍA}

- Atxtegui J. (2002) La depresión en los inmigrantes: una perspectiva transcultural. Ediciones Mayo, Barcelona.

- Gentil I (2006) Enfermería y personas de otras culturas. Cultura de los Cuidados, n 19, 37-40.

- Giger J, Davidizar R, Purnell L, Taylor Harden J, Phillips J, Strickland (2007) American Academy of Nursing Expert Panel Report: Developing Cultural Competence to Eliminate Health Disparities in Ethnic Minoritie and other Vulnerable Populations. Journal of Trascultural Nursing 18 (2), 95-102.

- Herman C. (2007) Culture, Health and illnes. Fifth Edition. Odre Arnold, London.

- Leininger M. (1999) Cuidar a los que son de culturas diferentes requiere el conocimiento y las aptitudes de la enfermería transcultural. Cultura de los Cuidados 6, 5-8.

- MTI. Ministerio de Trabajo e Inmigración. Secretaria de Estado de Inmigración y Emigración. Datos estadísticos a 610-2009. Disponible en http://extranjeros.mtin.es/es/ InformacionEstadistica/Anuarios/ [Consultado 19 febrero 2010]

- Nogales A. (2008) La empatía según Edith Stein. Cultura de los Cuidados 24, 119-133.

- Pascual Martínez R. et al., (2001) Pensamiento mágico y religiosidad en torno a la enfermedad: Manifestaciones simbólicas y rituales; función y significado. Cultura de los Cuidados 9, 49-62.

- Siles J. et al. (2001) Una mirada a la situación científica de dos especialidades esenciales de la enfermería contemporánea: la antropología de los cuidados y la enfermería transcultural. Cultura de los Cuidados 10, 72-87. 